\title{
Diffusion equation under swelling stresses
}

\author{
K. G. Schell ${ }^{1}$ (1) $\cdot$ T. Fett $^{1}$ - E. C. Bucharsky ${ }^{1}$
}

(c) Springer Nature Switzerland AG 2019

\begin{abstract}
Water diffusing into silica glass surfaces reacts with the $\mathrm{SiO}_{2}$ structure under hydroxyl generation. This reaction must cause a volume expansion that is already reported in literature. As a consequence of volume swelling and restriction of free expansion by the bulk material, swelling stresses are caused. Since the diffusivity is a function of stress, the consequence is a diffusivity that depends on the local water concentration. Then the solution of the diffusion equation is complicated and makes numerical computations necessary. Disadvantage of numerical computations is the fact that the used extend of the depth range must be finite and, consequently, the semi-infinite body can only be approximated. In the following considerations we will discuss analytical diffusion solutions for constant diffusivity as well as under swelling conditions. Based on this, exact and semi-analytical solutions for diffusion problems in the half-space are given. Further, the effect of swelling affected diffusivity on resulting concentration profiles is shown. We used an analytical solution of the diffusion problem that is known from literature by Gardner and Singh, where the concentration-dependent diffusivity had to be approximated by an exponential function. In case of swelling stresses in silica the diffusivity is given by an exponential function, so that the obtained solution is exact. Due to the compressive swelling stresses, steeper diffusion profiles appear compared to the stress-free state. In the discussion section it will be shown that the water-affected diffusivity correctly predicts the astonishing time-dependency of effective diffusivities observed in literature on silica.
\end{abstract}

Keywords Diffusion equation · Silica/water reaction · Residual stresses · Stress affected diffusion · Saturation profiles

\section{Introduction}

The partial diffusion differential equation for the uniaxial case is

$\frac{\partial C}{\partial t}=\frac{\partial}{\partial z}\left[D(C) \frac{\partial C}{\partial z}\right]$

Here $C$ is the water concentration, $t$ the time, $z$ the depth coordinate, and $D$ the diffusivity that may depend on the water concentration.

For water vapour as the environment, the surface condition is

$\frac{d C}{d z}=\frac{h}{D}\left(C-C_{0}\right)$ at $z=0$, where $C_{0}$ is the concentration of molecular water reached at $z=0$ for $t \rightarrow \infty$.

Following the suggestion by Doremus ([1], Section 4.7), the phenomenological parameter $h$ in (2) may be interpreted as a reaction parameter for a slow surface reaction that limits the entrance of molecular water species.

On the other hand, a simpler phenomenological description is possible by assuming that a barrier exists to the transport of water across the surface of the glass. The barrier gives rise to a mass transfer coefficient for diffusion, which slows the passage of water into the glass [1]. Each of the assumptions yields the same set of mathematical equations.

The Eqs. (1) and (2) can be solved numerically as was done in [2]. Disadvantage of numerical computations is the fact that the used extend of the $z$-range must be

K. G. Schell, g.schell@kit.edu | ${ }^{1}$ Karlsruhe Institute of Technology, Institute for Applied Materials, Karlsruhe, Germany.

SN Applied Sciences (2019) 1:1300 | https://doi.org/10.1007/s42452-019-1343-1

Received: 5 August 2019 / Accepted: 24 September 2019 / Published online: 28 September 2019 
finite and, consequently, the semi-infinite body can only be approximated. In the following considerations we will give exact and approximate solutions of the diffusion differential Eq. (1) for the half-space.

The problems mentioned here can be solved by "classical" approaches. For much more complicated problems, more specific methods are needed. For example, diffusion is treated in disordered materials by Giona and Roman in [3]. In this reference a solution is analytically obtained for diffusion on fractals proposed for materials showing anomalous transport behavior.

The reason for the concentration-dependent diffusivity used here is the hydroxyl generation at the silica surface exposed to humid environments. Water penetrated into silica reacts with the silica network according to [4]

$\equiv \mathrm{Si}-\mathrm{O}-\mathrm{Si} \equiv+\mathrm{H}_{2} \mathrm{O} \leftrightarrow \equiv \mathrm{SiOH}+\mathrm{HOSi} \equiv$

with the concentration of the hydroxyl $S=[\equiv \mathrm{SiOH}]$ and that of the molecular water $C=\left[\mathrm{H}_{2} \mathrm{O}\right]$.

A swelling effect in water-containing silica at high temperatures was early reported by Brückner [5, 6], Shackelford [7] and Shelby [8]. These authors showed that the density decreases by the silica/water-reaction. This decrease is equivalent to a volume expansion $\varepsilon_{\mathrm{v}} \approx 0.97 \mathrm{~S}$ [2], i.e. $\varepsilon_{\mathrm{v}} \propto C$.

\section{Analytical solution of the diffusion equation for constant diffusivity}

First, let us consider the case of constant diffusivity, $D=D_{0}$. As shown by Carslaw and Jaeger ([9], Section 2.7), the concentration profile, $C(z)$ resulting from the boundary condition for a semi-infinite body is given by

$\frac{C(\zeta, \tau)}{C_{0}}=\operatorname{erfc}\left[\frac{\zeta}{2}\right]-\exp [\zeta \sqrt{\tau}+\tau] \operatorname{erfc}\left[\frac{\zeta}{2}+\sqrt{\tau}\right]$

At the surface, $z=0$ :

$C(0, \tau) / C_{0}=1-\exp [\tau] \operatorname{erfc}[\sqrt{\tau}]$,

with the normalized dimensionless time $\tau$ and normalized depth coordinate $\zeta$, defined by

$\tau=\frac{h^{2}}{D_{0}} t ; \zeta=\frac{z}{\sqrt{D_{0} t}}$

For the ratio $C(\zeta, \tau) / C(0, \tau)$

$\frac{C(\zeta, \tau)}{C(0, \tau)}=\frac{\operatorname{erfc}\left[\frac{\zeta}{2}\right]-\exp [\zeta \sqrt{\tau}+\tau] \operatorname{erfc}\left[\frac{\zeta}{2}+\sqrt{\tau}\right]}{1-\exp [\tau] \operatorname{erfc}[\sqrt{\tau}]}$

two limit cases are of special interest. At very short times, $\tau \rightarrow 0$, we obtain by a series expansion with respect to $\tau$

SN Applied Sciences

A SPRINGER NATURE journal
$\frac{C(\zeta, 0)}{C(0,0)}=\exp \left[-\frac{\zeta^{2}}{4}\right]-\frac{\sqrt{\pi}}{2} \zeta \operatorname{erfc}\left[\frac{\zeta}{2}\right]$

At very long times $\tau \rightarrow \infty$, only the first term on the right-hand side of Eq. (3) remains finite. Consequently, we obtain the well-known solution for constant surface concentration:

$\frac{C(\zeta, \infty)}{C(0, \infty)}=\operatorname{erfc}\left[\frac{\zeta}{2}\right]$

These limit cases are plotted in Fig. 1a. The depths at which these limit distributions decrease to $C(\zeta, \tau) / C(0, \tau)=1 / 2$ are

$\zeta_{1 / 2}=0.6995 \quad$ for $\quad \tau \rightarrow 0$

$\zeta_{1 / 2}=0.9538$

for $\tau \rightarrow \infty$

The areas under the curves define the water uptake in normalized time and depth units

$m_{C}(\tau)=\int_{0}^{\infty} C(\zeta, \tau) d \zeta=C(0, \tau) \times \begin{cases}\frac{\sqrt{\pi}}{2} \text { for } & \tau \rightarrow 0 \\ \frac{2}{\sqrt{\pi}} \text { for } & \tau \rightarrow \infty\end{cases}$

For etching tests it is of advantage to know the amount of water $\Delta m_{C}$, when a layer of thickness $\delta$ has been removed from the surface. In this case it holds

$$
\begin{aligned}
& \Delta m_{C}(\delta, \tau)=-\int_{0}^{\delta} C(\zeta, \tau) d \zeta= \\
& =-m_{C}(\tau) \begin{cases}\frac{1}{\sqrt{\pi}} \delta \exp \left[-\frac{1}{4} \delta^{2}\right]+\operatorname{erf}\left[\frac{1}{2} \delta\right]-\frac{1}{2} \delta^{2} \operatorname{erfc}\left[\frac{1}{2} \delta\right] & \text { for } t \rightarrow 0 \\
\left(1-\exp \left[-\frac{1}{4} \delta^{2}\right]\right)+\frac{\sqrt{\pi}}{2} \delta \operatorname{erfc}\left[\frac{1}{2} \delta\right] & \text { for } t \rightarrow \infty\end{cases}
\end{aligned}
$$

The results from (11) are shown in Fig. 1b. For the thickness removal $d$ in normal thickness unit we have to replace $\delta$ by $d / \sqrt{ }\left(D_{0} t\right)$.

\section{Solutions under swelling conditions}

\subsection{Stress enhanced diffusion}

The diffusivity as a function of stress is commonly expressed by the hydrostatic stress component, $\sigma_{\mathrm{h}}$. The 
Fig. 1 a Concentration profiles for limit cases derived from the analytical solution of diffusion, Eqs. $(7,8)$ with constant diffusivity, $D=D_{0}$. b Water uptake according to Eq. (11). The negative sign at $\Delta m_{C}$ stands for the decrease of the water content (a)

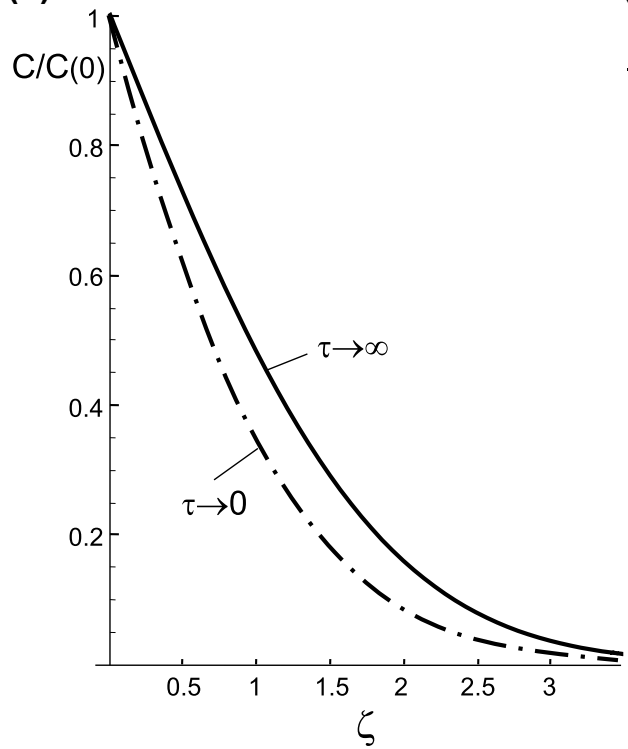

(b)

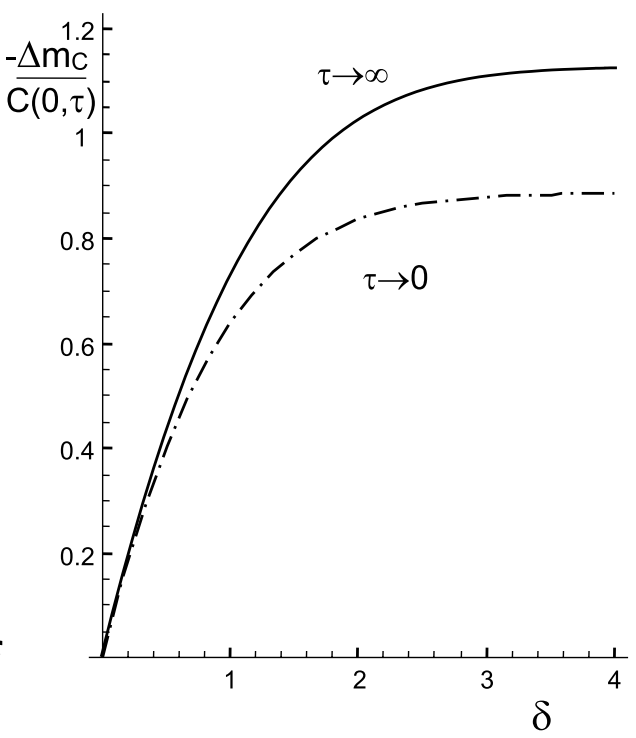

diffusivity for the case of stress-enhanced diffusion is given by the following equation [10]

$D=D_{0} \exp \left[\sigma_{h} \frac{\Delta V_{w}}{R T}\right]$

where $D_{0}$ denotes the value of the diffusivity in the absence of a stress. $T$ is the absolute temperature in $K ; \Delta V_{w}$ is the activation volume for stress-enhanced diffusion and $R$ is the universal gas constant.

The hydrostatic stress term caused by swelling stresses is

$\sigma_{h}=-\frac{2 \kappa E}{9(1-v)} k C, \quad \kappa \cong 0.97$,

where $E$ is Young's modulus, $v$ Poisson's ratio, and $k$ is the equilibrium constant of the silica/water reaction given for temperatures $<500^{\circ} \mathrm{C}$ by $k=S / C(C=$ molecular water concentration, $S=$ hydroxyl concentration).

According to Eq. (13) the swelling stress depends linearly on the water concentration, $\sigma_{\mathrm{h}} \propto C$. The saturation value of $\sigma_{\mathrm{h}, \mathrm{sw}}$ for $C=C_{0}$ is in the following considerations denoted as $\sigma_{h, 0}$. In order to allow short expressions, the exponential term in Eq. (12) may be abbreviated by

$\sigma_{h} \frac{\Delta V_{w}}{R T}=\sigma_{h, 0} \frac{\Delta V_{w}}{R T} \frac{C}{C_{0}} \equiv \alpha C, \quad \alpha \equiv \frac{\sigma_{h, 0}}{C_{0}} \frac{\Delta V_{w}}{R T}$

\subsection{Solution based on a perturbation set-up by Singh}

By use of the Boltzmann substitution

$\lambda=\frac{z}{2 \sqrt{t}} \Rightarrow \lambda=\sqrt{D_{0}} \frac{\zeta}{2}$ an ordinary differential equation results from Eq. (1)

$2 \lambda \frac{d C}{d \lambda}+\frac{d}{d \lambda}\left[D \frac{d C}{d \lambda}\right]=0 \Rightarrow \zeta \frac{d C}{d \zeta}+2 \frac{d}{d \zeta}\left[\frac{D}{D_{0}} \frac{d C}{d \zeta}\right]=0$

Gardner [11] and later Singh [12] showed that this equation can be solved if the diffusion coefficient fulfills an exponential relation

$D=\exp (\alpha C+\beta)$

with constant coefficients $a$ and $\beta$. This result is used in bottom mechanics [13] where the diffusivity depends on the water concentration, too.

The solution based on a perturbation ansatz reads

$D=c_{1} \frac{\sqrt{\pi D_{0}}}{2} \operatorname{erf} \frac{\zeta}{2}+c_{2}$

For the swelling problem the condition (17) is fulfilled since

$D=D_{0} \exp \left(\Delta V_{w} \sigma_{h} / R T\right)=D_{0} \exp (\alpha C)$,

$\beta=\ln D_{0}, \alpha C=\frac{\Delta V_{w} \sigma_{h}}{R T}$

Combining Eqs. (18) and (19) yields

$c_{1} \frac{\sqrt{\pi D_{0}}}{2} \operatorname{erf} \frac{\zeta}{2}+c_{2}=D_{0} \exp (\alpha C)$

and from this the water concentration results as a function of depth $z$ and time $t$

$C=\frac{1}{\alpha} \ln \left(c_{1} \frac{\sqrt{\pi / D_{0}}}{2} \operatorname{erf} \frac{\zeta}{2}+\frac{c_{2}}{D_{0}}\right)$ 
For $z \rightarrow \infty$ it must hold $C \rightarrow 0, D \rightarrow D_{0}$. This condition gives with $\operatorname{erf}[\infty]=1$

$c_{1} \frac{\sqrt{\pi / D_{0}}}{2}+\frac{c_{2}}{D_{0}}=1 \Rightarrow c_{2}=D_{0}-c_{1} \frac{\sqrt{\pi D_{0}}}{2}$

Replacing $c_{2}$ in Eq. (22) results in the solution

$C=\frac{1}{\alpha} \ln \left(c_{1} \frac{\sqrt{\pi / D_{0}}}{2} \operatorname{erf} \frac{\zeta}{2}+1-c_{1} \frac{\sqrt{\pi / D_{0}}}{2}\right)$

or with the complementary error function $\operatorname{erfc}(x)=1-\operatorname{erf}(x)$ :

$C=\frac{1}{\alpha} \ln \left(1-c_{1} \frac{\sqrt{\pi / D_{0}}}{2} \operatorname{erfc} \frac{\zeta}{2}\right)$

\subsubsection{Increasing surface concentration (mass-transfer condition)}

A solution for the surface conditions by Eq. (2) cannot result from Singh's procedure. This can even be seen from the application of the Boltzmann substitution. In terms of the normalized time and depth coordinate by (5), the substitution $\lambda$ in Eq. (15) is only dependent on the depth coordinate $\zeta$ and not the time $\tau$. Consequently the applicability to time-dependent diffusion effects given by Eq. (2) is strongly restricted. Nevertheless, this solution is appropriate for treating the limit case $\tau \rightarrow \infty$, i.e. for the condition of fixed surface concentrations.

\subsubsection{Constant surface concentration}

For very long diffusion times, the surface water concentration, $C(0)$, tends asymptotically to the saturation value $C_{0}$. In order to compute the limit case for $t \rightarrow \infty$. Specimen soaked in water vapour for very long times are assumed to show constant surface water concentration $C(z=0)=C_{0}$. In this case we obtain from (25)

$C_{0}=\frac{1}{\alpha} \ln \left(1-c_{1} \frac{\sqrt{\pi / D_{0}}}{2}\right)$

the constant $c_{1}$ as

$c_{1}=2 \sqrt{D_{0} / \pi}\left(1-\exp \left[\alpha C_{0}\right]\right)$

The result for the concentration is then

$\frac{C}{C_{0}}=\frac{1}{\alpha C_{0}} \ln \left(1-\left(1-\exp \left[\alpha C_{0}\right]\right) \operatorname{erfc}\left[\frac{\zeta}{2}\right]\right)$

Water profiles computed via Eq. (28) are shown in Fig. $2 a$ for different parameters $a C_{0}$. Figure $2 b$ shows a comparison of the analytical solution Eq. (28) as the black curve and the numerical results according to [2] as the red curve, both for $a C_{0}=-3$. The small differences may be the consequence of the finite depth interval of $5 \times \delta_{1 / 2}$ (i.e. the concentration boundary condition at this location prescribed by $C\left(\zeta=5 \times \delta_{1 / 2}\right)=0$ ) that had to be used in the numerical program NDSolve by Mathematica [14].

The depth $\zeta_{1 / 2}$ at which the distributions of Fig. $2 \mathrm{a}$ decreases to $C(\zeta) / C_{0}=1 / 2$ is
Fig. 2 a Effect of swelling on the water profiles, $\mathbf{b}$ comparison of Eq. (28) with numerical solution from [2], given by the black and red curve, respectively (a)

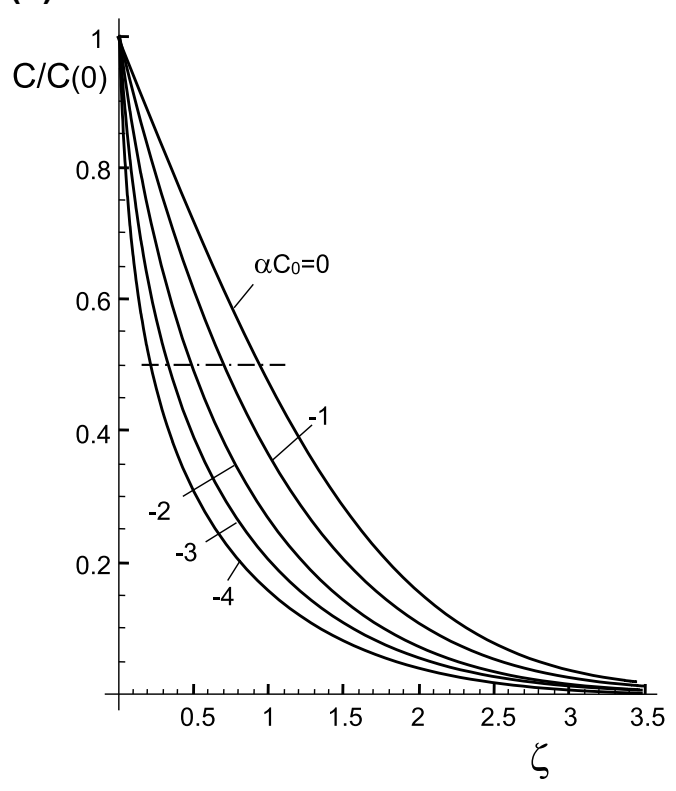

(b)

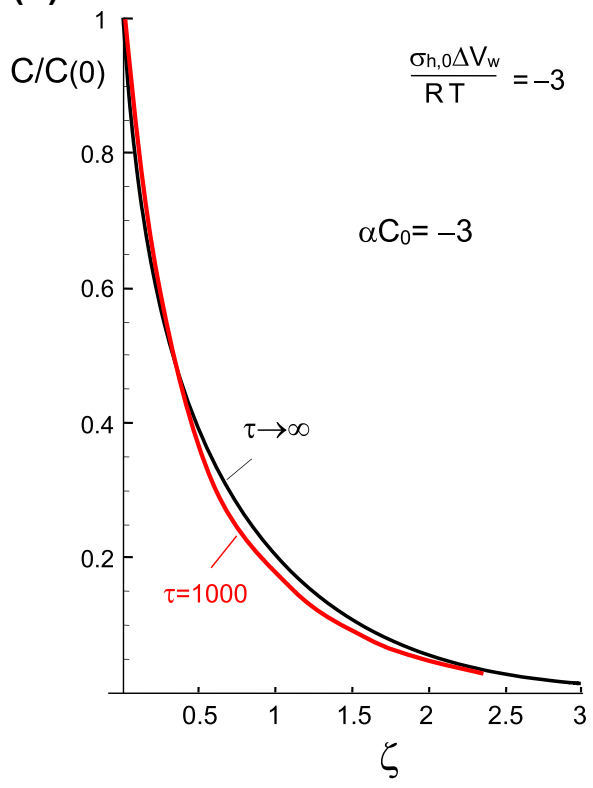


Table 1 Data of water profiles and water uptake obtained for saturation conditions, $\tau \rightarrow \infty$

\begin{tabular}{llll}
\hline $\mathrm{a} C_{0}$ & $\zeta_{1 / 2}=z_{1 / 2} / \sqrt{ } D_{0} \tau$ & $m_{\mathrm{C}}(\tau) / C(0)$ & $\delta_{1 / 2}=d_{1 / 2} / \sqrt{ } D_{0} \tau$ \\
\hline 0 & 0.9538 & $2 / \sqrt{ } \pi$ & 0.6994 \\
-1 & 0.6963 & 0.9115 & 0.6036 \\
-2 & 0.4861 & 0.7319 & 0.5323 \\
-3 & 0.3262 & 0.5904 & 0.4846 \\
-4 & 0.2121 & 0.4823 & 0.4558 \\
\hline
\end{tabular}

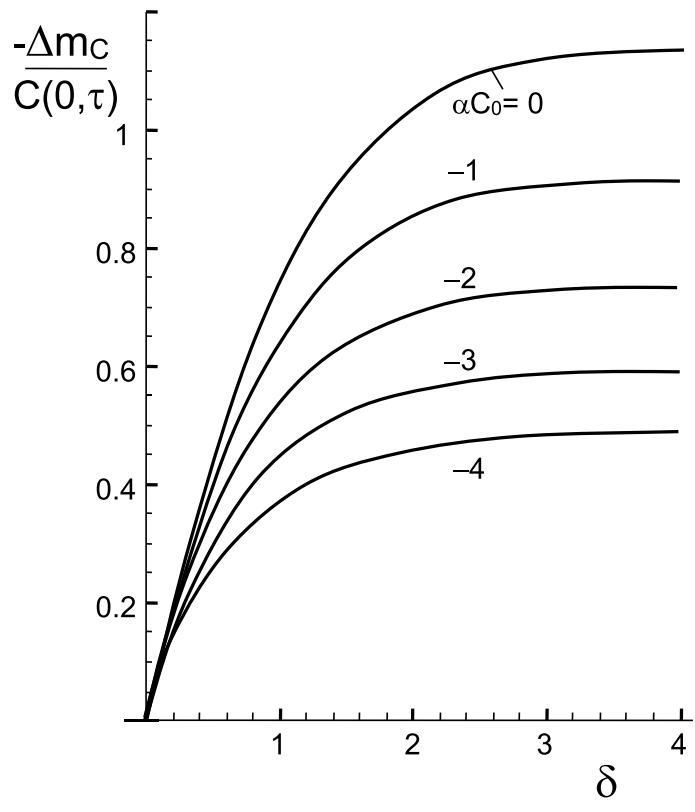

Fig. 3 Change of water uptake with normalized surface removal depth $\delta$ as a function of swelling parameter $a C_{0}$ normalized on the uptake for $a C_{0}=0$

$\zeta_{1 / 2}=2 \operatorname{erf}^{-1}\left[\infty,-\frac{1}{1+\exp \left[\frac{1}{2} \alpha C_{0}\right]}\right]$

Numerical values are compiled in the second column of Table 1.

Finally, we determined water uptake by integrating the swelling profiles of Fig. 2a numerically with the result

$m_{C}(\tau)=\int_{0}^{\infty} C(\zeta, \tau) d \zeta$

with results given in the third column of Table 1 for $\tau \rightarrow \infty$. The value for $a C_{0}=0$ is $2 / \sqrt{ } \pi \cong 1.128$ as given by Eq. (10).

The decrease of the water by surface removal $d$ is shown in Fig. 3 in terms of the normalized quantity $\delta$
$\delta=\frac{d}{\sqrt{D_{0} t}}$

The depths at wich half of the water content is removed, $\delta_{1 / 2}$, is given in the fourth column of Table 1 for a few values of $a C_{0}$.

\section{Discussion}

Results of computations are shown in Fig. $4 a$, b. The bold curve in Fig. 4a a is the limit case for saturation conditions for $\tau \rightarrow \infty$, given by Eq. (25) and the thin curves represent the numerical solution of Eqs. (1) and (2). The widths of the profiles allow an effective diffusivity to be determined according to Davis and Tomozawa [15]

$C=C_{0} \operatorname{erfc}\left(\frac{z}{2 \sqrt{D_{\text {eff }} t}}\right)$

In [15] the effective diffusivity, $D_{\text {eff }}$ is determined from the width at $C / C_{0}=1 / 2$ interpreting it as the diffusion lengths $\sqrt{D_{\text {eff }} t}$. This effective diffusivity $D_{\text {eff }}$ is usually determined in investigations on water diffusion in silica [15-18].

Measurements of effective diffusivities by Oehler and Tomozawa [16] at $250^{\circ} \mathrm{C}$ and 39 bar water vapour pressure are introduced by circles, see Fig. 4c. The curve is introduced as a guideline for the eyes. A clear decrease with time is evident. This effect was discussed in [2] in terms of swelling stresses.

\section{Summary}

Water present at silica surfaces reacts with the silica network under generation of hydroxyl that causes a volume expansion of the thin water-affected zone. Since free expansion is restricted by the bulk material, compressive swelling stresses result which yield in a reduced diffusivity. Stress affected diffusion was considered and resulting saturation profiles were derived by the analytical procedure of Gardner [11] and Singh [12]. The calculations show that the compressive swelling stresses result in steeper profiles caused by a decreased diffusivity compared to stress-free state. In order to show the occurrence of reduced diffusivity, we compared the predicted water profiles with measurements by Öhler and 
Fig. 4 a Diffusion profiles as a function of time without and with consideration of swelling stress effect on diffusivity (thin curves computed numerically, bold curves: analytical solutions), $\mathbf{b}$ effective diffusivity $D_{\text {eff }}$ vs. normalized time $\tau$, c effective diffusivities measured by Oehler and Tomozawa [16] at $250^{\circ} \mathrm{C}$ and 39 bar water vapour pressure (a)

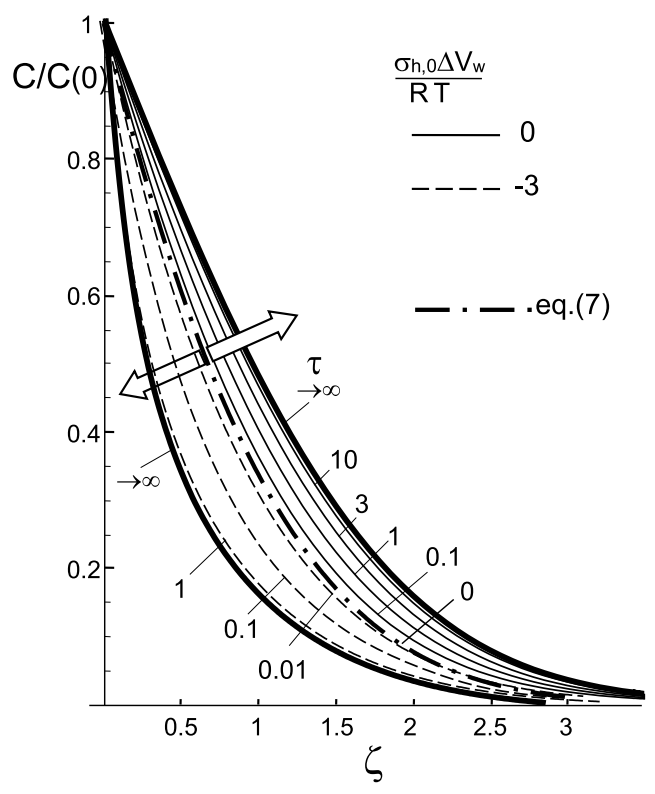

(b)

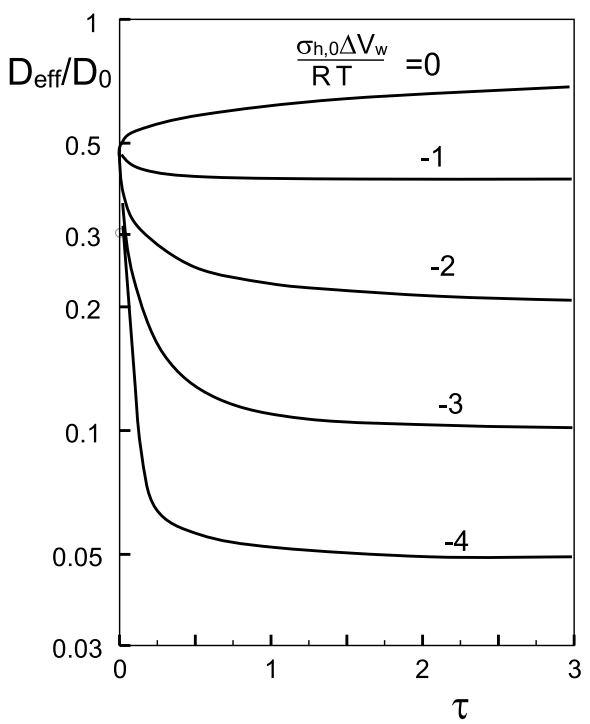

(c)

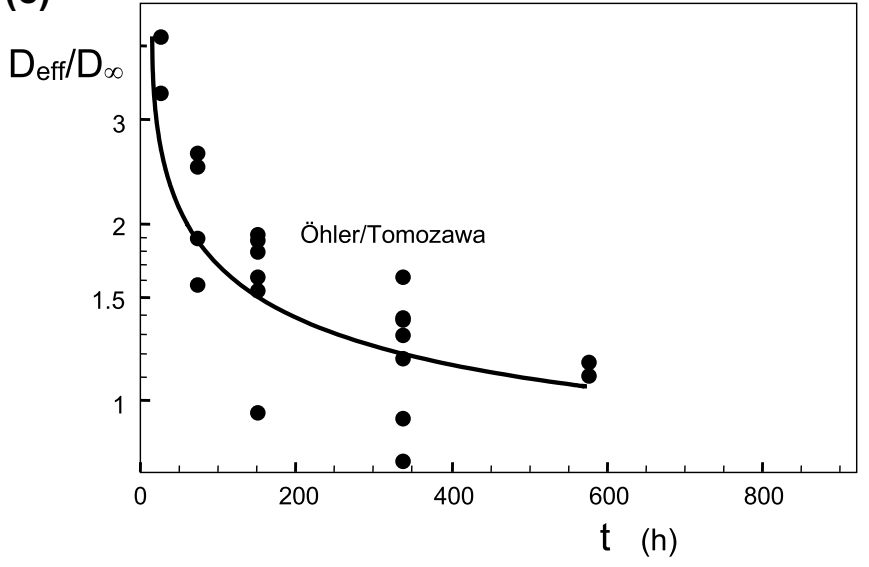

Tomozawa [16]. The predictions made by computations show the same tendency as experimental results from literature which couldn't be interpreted sufficiently so far.

\section{Compliance with ethical standards}

Conflict of interest The authors declare that they have no conflict of interest.

\section{References}

1. Doremus RH (2002) Diffusion of reactive molecules in solids and melts. Wiley, New York

2. Wiederhorn SM, Rizzi G, Wagner S et al (2017) Diffusion of water in silica: influence of moderate stresses. J Am Ceram Soc. https ://doi.org/10.1111/jace.15195
3. Giona M, Roman HE (1992) A theory of transport phenomena in disordered systems. Chem Eng J 49:1-10. https://doi. org/10.1016/0300-9467(92)85018-5

4. Doremus RH (1995) Diffusion of water in silica glass. J Mater Res 10:2379-2389. https://doi.org/10.1557/JMR.1995.2379

5. Brückner R (1970) The structure-modifying influence of the hydroxyl content of vitreous silica. Glas Ber 43:8-12

6. Brückner R (1971) Metastable equilibrium density of hydroxylfree synthetic vitreous silica. J Non Cryst Solids 5:281-285. https ://doi.org/10.1016/0022-3093(71)90068-8

7. Shackelford JF, Masaryk JS, Fulrath RM (1970) Water content, fictive temperature, and density relations for fused silica. J Am Ceram Soc 53:417. https://doi.org/10.1111/j.1151-2916.1970. tb12145.x

8. Shelby JE (2004) Density of vitreous silica. J Non Cryst Solids 349:331-336. https://doi.org/10.1016/j.jnoncrysol.2004.08.206

9. Carslaw HS, Jaeger JC (1959) Conduction of heat in solids, 2nd edn. Oxford at the Clarendon Press, London

10. Shewmon PG (1963) Diffusion in solids. McGraw-Hill, New York

11. Gardner WR (1958) Some steady-state solutions of the unsaturated moisture flow equation with application to 
evaporation from a water table. Soil Sci 85:228-232. https:// doi.org/10.1097/00010694-195804000-00006

12. Singh R (1967) Solution of a diffusion equation. J Hydraul Div Proc Am Soc Civ Eng 93(5):5422-5450

13. Boochs PW, Battermann G, Mull R (1972) Abhängigkeit des Diffusionskoeffizienten für Wasser vom Sättigungsgrad des Bodens. Zeitschrift für Pflanzenernährung und Bodenkd 132:243-253. https://doi.org/10.1002/jpln.19721320309

14. Wolfram Research I (2016) Mathematica

15. Davis KM, Tomozawa M (1995) Water diffusion into silica glass: structural changes in silica glass and their effect on water solubility and diffusivity. J Non Cryst Solids 185:203-220. https://doi. org/10.1016/0022-3093(95)00015-1
16. Oehler A, Tomozawa M (2004) Water diffusion into silica glass at a low temperature under high water vapor pressure. J Non Cryst Solids 347:211-219. https://doi.org/10.1016/j.jnoncrysol .2004.07.013

17. Zouine A, Dersch O, Walter G, Rauch F (2007) Diffusivity and solubility of water in silica glass in the temperature range $23-200^{\circ} \mathrm{C}$. Phys Chem Glass Eur J Glass Sci Technol Part B 48:85-91

18. Helmich M, Rauch F (1993) On the mechanism of diffusion of water in silica glass. Glasstech Ber 66:195-200

Publisher's Note Springer Nature remains neutral with regard to jurisdictional claims in published maps and institutional affiliations. 